\title{
NATURAL VALORIZATION OF DEGRADED SWAMP-ORIGINATING MEADOWS OF LOWER SILESIA (EXTENSION OF THE OŚWIT METHOD)
}

\author{
Magda Podlaska \\ M. Podlaska, Department of Botany and Plant Ecology, Wrocław University of Environmental and Life \\ Sciences, pl. Grunwaldzki 24 a, 50-363 Wrocław, Poland, e-mail: magda.podlaska@up.wroc.pl
}

(Received: January 9, 2014. Accepted: March 31, 2014)

\begin{abstract}
AвSTRACT. Swamp-originating meadows constituting indirect habitats between living peatlands and classical grasslands may be difficult to evaluate. An indiscriminate application of the OśwIT (2000) method for their valorization does not fully reflect their value, because of flora which is different from flora found in living peatlands (in particular, the invasion of less hygrophilous flora). Based on the research carried out in the years 2005-2010 on eight swamp-originating meadow complexes, the scale was elaborated which enabled to more accurately valorize these habitat and floristically changed areas. The quantities of valorization values obtained by use of the author's method rely on the occurrence frequency of a given species (a number of posts in a given area), taking into consideration moisture requirements (the moisture indicator). In the author's method values were also given for species not taken into account by OśwIT (2000). In addition, positive points were given for dying out species, and negative points for invasive species.
\end{abstract}

KEY WORDS: swamp-originating meadows, valorization, the Oświt method, the author's method

\section{INTRODUCTION}

Peatlands as ecosystems of complicated and fragile balance are very sensitive and respond violently to all kinds of changes. Major factors conditioning the transformation and disappearance of peatlands are inappropriate meliorations (grassland improvements) and an inadequate management and use of them (ILNICKI 2002, ToвolsKi 2003).

Relatively recently, attention was drawn to the changes in the species composition of plant communities of low peatlands as well as swamp-originating meadows which appeared after their drainage. The occurring transformations were analysed in the context of swamp-originating meadows degradation and its influence on the yield of grasslands (ProŃczuk 1956, ОкRUSzKo 1966), and on the natural environment (NorYśkIEWICZ 1978, GrYNIA 1980), as well as in respect of floristic and ecological issues (JASNOWSKI 1972, PAŁCZYŃSKI 1977, 1985). After the transformation of economic conditions in Poland, many swamp-originating meadows were put out of use or their use was dramatically limited, simultaneously ecognizing changes occurred in meadow communities (ILNICKI et al. 2004, KozŁowsKa 2005), while each area took its own direction of transformations which resulted in a diversity of the natural value.

The hitherto valorization methods of marshlands referred mainly to living and well-maintained peatlands (OśwIT 2000), however they were not suitable for so greatly transformed sites as swamp-originating meadows.

This paper constitutes an extension and completion of the Oświt (2000) method used for the valorization of marshes, in order to adopt it for the conditions prevailing in degraded swamp-originating meadows.

\section{MATERIALS AND METHODS}

Swamp-originating meadows are defined as lowland meadows included in the group of swamp meadows, occurring in artificially drained and drying up podsol, as well as pool and still water marshy meadows. The soils of these meadows are muck (peat and mud-organic soils subject to the process of rotting), 
of variable moisture conditions, and the water table is always found beneath the depth of $50 \mathrm{~cm}$.

\section{FIELD EXAMINATIONS}

The floristic and phytosociological research was carried out in the selected swamp-originating meadows in 2005-2010. The meadows selected for this research are situated in the lowland area of the Lower Silesia and are called as follows: Kotla, Głogówko, Parowa, Brnowiec, Przedmoście, Miękinia I and II as well as Milicz. They are meadow complexes of the area above 30 ha and documented farm use (PAECZYŃSKI 1970, 1976, Stepa 1976, KalbarczyK 1982, SobCZaK 1982, BADANiA... 1983 a, b, KowalsKa 1990), which came into existence at the beginning of the 20th century as a result of melioration of lowland peatlands. Initially, they were used relatively intensively as meadows and pastures and they were characterised by adequate, from the viewpoint of a meadow expert, water relations. At present, they are not in use or are used extremely extensively, and the moisture conditions prevailing there are very changeable. However, they are valuable areas especially for the transformed agricultural landscape.

The plant species were denominated by use of the keys of Rothmaler (2002) and RUtKOWSKI (2004). The names of vascular plant species were adopted after Mirek et al. (2002). The synthaxonomic affiliation was defined according to Matuszkiewicz (2006), Kucharski \& Michalska-Hejduk (1994), Pott (1995) as well as KUCHARSKI et al. (2001).

\section{ANALYSIS OF NATURAL VALUES}

The natural value of plant communities was evaluated by use of the method elaborated by Oświt (2000) and by the author's method. The Oświt method involved assigning a particular numerical value (from 1 to 10 ) to respective species occurring in phytocoenoses, and then calculating the mean value for each phytocoenosis (the phytosociological picture) by adding up the above values for all the obtained taxa and dividing the acquired sum by the total number of species in a community (the picture). The obtained results represented the valorization class to which the natural value of a community was assigned (Table 1).

The data analysis by use of the OśwIT (2000) method has indicated that it may not be indiscriminately applied for describing the degraded swamp-originating meadows of southwestern Poland, as it is limited in such respects as follows:

- the Oświr (2000) method does not include many species of vascular plants which are found in the swamp-originating meadows of Lower Silesia, such as hygrophilous taxa;

- it presents a different geobotanical specificity of Lower Silesia (presence of subatlantic species, fewer taxa typical for areas of a higher degree of continentalism; lack of supraregional valorization character);

- it includes the overestimation of values for species more frequently occurring in southern and southwestern Poland, e.g. Lathyrus pratensis;

- the Oświt (2000) elaboration requires to be verified each time as there have appeared a new list of protected species (RozPORZĄDZENIE... 2004, 2012). In order to evaluate the natural value of rare species it is essential to become acquainted with the regional lists of endangered plants (in reference to the examined objects e.g. КĄскI 2003);

- it includes relict species, which owing to their extremely scarce occurrence in Poland, do not constitute a measurable valorization indicator.

A critical analysis of the OśwIt (2000) method resulted in the author's proposition of natural valorization of swamps, in particular of the swamp-originating meadows of southwestern Poland. The following

Table 1. Valorization classes for marshlands and hydrogenic habitats according to the Oświt method and the author's method (southwestern Poland) (according to: Oświt 2000)

\begin{tabular}{clcc}
\hline \multirow{2}{*}{ Valorization classes } & \multicolumn{2}{c}{ Description of natural values } & \multicolumn{2}{c}{ Mean valorization indicator } \\
\cline { 3 - 4 } I & very low natural values & Oświt method & author's method \\
\hline II & averagely low natural values & $<1.4$ & 2.00 \\
III & low natural values & $1.5-1.8$ & $2.00-3.19$ \\
IV & moderate natural values & $1.9-2.2$ & $3.20-4.39$ \\
V & averagely moderate natural values & $2.3-2.6$ & $4.40-5.59$ \\
VI & moderately high natural values & $2.7-3.0$ & $5.60-6.79$ \\
VII & high natural values & $3.1-3.4$ & $6.80-7.99$ \\
VIII & very high natural values & $3.5-3.8$ & $8.00-9.19$ \\
IX & outstanding natural values & $3.9-4.2$ & $9.20-10.39$ \\
X & unique, exceptional natural values & $4.3-4.6$ & $10.40-11.59$ \\
\hline
\end{tabular}


valorization criteria (which, according to the author, are more objective and concise) were adopted:

- evaluation was carried out exclusively on the regional scale for the Silesian Lowland and Sasko-Łużycka Lowland;

- it was assumed that all the non-cultivated plant species found in the examined swamp-originating meadows are taxa occurring there spontaneously (sponateophytes), whose ecological amplitude allows them to occupy this type of hydrogenic habitats;

- consideration was given to the habitat preferences of respective species expressed by the soil moisture indicator $(\mathrm{W})$, according to ZARZYCKI et al. (2002), which was converted into the 12-degree scale of the ELLENBERG (1979) indicator for moisture (Feuchtezahl - F), and then transformed in such a way as to obtain the value of zero (WF) for dry habitat plants ( $\mathrm{W}=2$ / $\mathrm{F}=3)$, as well as to make the differences between respective categories equal to 1 (Table 2). The mean values were adopted in case of the species of a wider hydrologic spectrum. Furthermore, due to significant discrepancies between the values of the moisture indicators given by ZARZYCKI et al. (2002) and ELLENBERG (1979) - for Geranium palustre the value of the $\mathrm{W}_{\mathrm{F}}$ indicator was decreased by 1 , and for $\mathrm{Hy}$ drocotyle vulgaris $\mathrm{W}_{\mathrm{F}}$ was increased by 1 . Despite the fact that Spiraea tomentosa, as a neophyte, was not included in either of the lists, it was taken into account by adopting the value of $\mathrm{W}_{\mathrm{F}}=4$ acquired in the course of the author's field observations;

- the occurrence frequency of all the obtained taxa of vascular plants was estimated according to the amount of their posts in the area, taking into account the number of ATPOL squares occupied by them (ZAJĄC \& ZAJĄC 2001), 264 squares were put to analysis. It was assumed that the valorization value (the number) of respective taxa was inversely proportional to the square root of the number of their posts according to "Distribution atlas of vascular plants in Poland" (ZAJĄC \& $\mathrm{ZA}_{\mathrm{A}}$ JĄC 2001) in the whole area, which is presented in Table 2. In case of Spiraea tomentosa, no data concerning the distribution of this species were found in "Distribution atlas..." in spite of its occurrence in Silesia as a spontaneophyte since the turning of the 19th and 20th centuries (SCHube 1903), where it was observed in the Dolnośląskie and Niemodlińskie Bory (Forests) among others. Based on this information as well as present-day observations (KRUKOWSKI M. oral information; TOKARSKA-GuzIK \& DAJDOK 2004), it was assumed that Spiraea tomentosa ocurs in at least 10 ATPOL squares;

- in addition, the valorization value was increased for endangered species under threat of fading out and extinction according to the category of their endangerment (КА̨скI 2003), adopting the following values: +4 for the EN category (Endangered) - Scutellaria hastifolia, +3 for the VU category (Vulnerable) - Dianthus superbus, Senecio congestus, +2 for the LC category (Least Concern) Isolepis setacea, Juncus bulbosus, and +1 for the NT category (Near Threatened) Lathyrus palustris, Ranunculus lingua, Thalictrum flavum;

- moreover, in case of strongly invasive non-native species (TokARsKa-GuziK \& DaJDOK 2004, Weber \& Gut 2004), the valorization value was decreased by -8 for: Impatiens parviflora, Solidago gigantea, Spiraea tomentosa, and by -4 for: Acorus calamus, Conyza canadensis, Fallopia convolvulus, Helianthus tuberosus, Oxalis fontana.

Lower plants and cultivated species were excluded from further analysis. The natural valorization value for each species is a sum of the above described components. For 27 species the numerical value was decreased, for 125 the value was increased, in 33 cases the value remained the same, for 50 species the value was added - the ones which were not included in the Oświt method (Podlaska 2009). The examples of valorization values calculated in such a way for vascular plants are presented in Table 2 . As a result of taking into account a negative biocenotic impact of invasive species, the foregoing indicators may also obtain negative values (Conyza canadensis, Fallopia convolvulus, Impatiens parviflora, Oxalis fontana, Solidago gigantea and Spiraea tomentosa).

In order to compare the statistical reliability of both methods (the valorization values according to Oświt (2000) and to the author's own method), they were analyzed by use of the Wilcoxon Matched-Pairs nonparametric test due to the lack of normality of the distribution of both data collections. The $\mathrm{H}_{0}$ zero hypothesis was as follows: the valorization values in both methods do not differ statistically (PODLASKA 2009). In both sets, 186 species were put to analysis which gave the test result of $Z=6.26063, p<0.001$. The obtained result allowed to reject the above hypothesis and to adopt an alternative one which demonstrated that the author's method differs from the Oświt (2000) method in respect of its approach to the natural valorization of marshlands.

In order to set the scope of divisions between the author's valorization classes of respective phytosociological pictures, the foregoing taxa of vascular plants were compared (186 species) by use of the values of basic statistics. The difference between the mean values of the valorization value of both lists amounted to 0.72 , and to 1.00 between their medians. It resulted in an extension of limit values of respective class divisions of phytosociological pictures by 0.9 in relation to the Oświt method. Furthermore, the author's method brought a solution to the lack of continuity, observed in the Oświt method, in respective valorization classes (systematically occurring 
Table 2. Way of calculating the valorization values in the author's method

\begin{tabular}{|c|c|c|c|c|c|c|c|c|c|}
\hline Species & 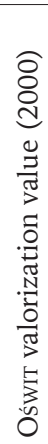 & 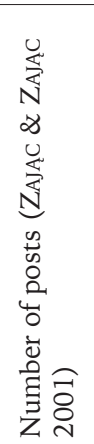 & 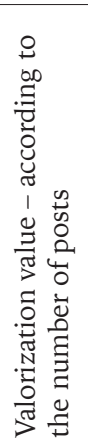 & 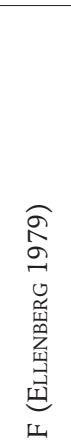 & 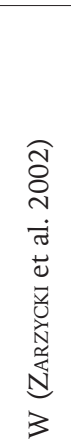 & $3^{4}$ & 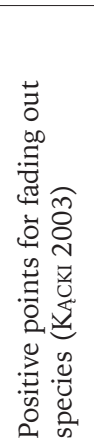 & 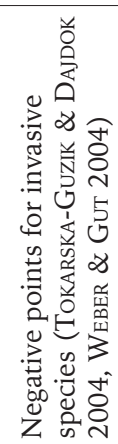 & 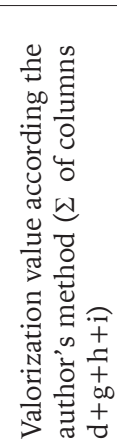 \\
\hline 1 & 2 & 3 & 4 & 5 & 6 & 7 & 8 & 9 & 10 \\
\hline Achillea millefolium & 2 & 263 & 1 & 4 & $2-3$ & -1 & & & 0 \\
\hline Achillea ptarmica & 8 & 94 & 3 & $8 \sim$ & 4 & 2 & & & 5 \\
\hline Acorus calamus & 4 & 191 & 2 & 10 & $5-6$ & 5 & & -4 & 3 \\
\hline Aegopodium podagraria & 2 & 260 & 1 & 6 & 3.5 & 1 & & & 2 \\
\hline Agrimonia eupatoria & 2 & 230 & 2 & 4 & $2-3$ & -1 & & & 1 \\
\hline Agrostis capillaris & 1 & 250 & 2 & $\mathrm{x}$ & $2-3$ & -1 & & & 1 \\
\hline Agrostis stolonifera & 4 & 220 & 2 & $6 \sim$ & 4 & 2 & & & 4 \\
\hline Alisma plantago-aquatica & 4 & 253 & 2 & 10 & $5-6$ & 5 & & & 7 \\
\hline Alliaria petiolata & 7 & 209 & 2 & 5 & 3.5 & 1 & & & 3 \\
\hline Alnus glutinosa & 4 & 260 & 1 & $9=$ & 5 & 4 & & & 5 \\
\hline Alopecurus geniculatus & 3 & 167 & 2 & $9=$ & 5 & 4 & & & 6 \\
\hline Alopecurus pratensis & 2 & 258 & 1 & 6 & 4 & 2 & & & 3 \\
\hline Anthoxanthum odoratum & 1 & 254 & 2 & $\mathrm{x}$ & 3 & 0 & & & 2 \\
\hline Apera spica-venti & . & 204 & 2 & 6 & 3 & 0 & & & 2 \\
\hline Arrenatherum elatius & 2 & 248 & 2 & 5 & 3 & 0 & & & 2 \\
\hline Avenula pubescens & 2 & 147 & 2 & $\mathrm{x}$ & $3-4$ & 1 & & & 3 \\
\hline Ballota nigra & . & 237 & 2 & 5 & 3 & 0 & & & 2 \\
\hline Berula erecta & 3 & 63 & 4 & $10 \sim$ & $6-5$ & 6 & & & 10 \\
\hline Betula pendula & . & 263 & 1 & $\mathrm{x}$ & 3 & 0 & & & 1 \\
\hline Betula pubescens & 3 & 105 & 3 & $\mathrm{x}$ & $4-5$ & 3 & & & 6 \\
\hline Bidens tripartita & . & 209 & 2 & $8=$ & $4-5$ & 3 & & & 5 \\
\hline Briza media & 1 & 214 & 2 & $\mathrm{x}$ & 3 & 0 & & & 2 \\
\hline Bromus hordeaceus & 1 & 207 & 2 & $\mathrm{x}$ & 3 & 0 & & & 2 \\
\hline Bromus inermis & 2 & 226 & 2 & $4 \sim$ & $2-3$ & -1 & & & 1 \\
\hline Bromus sterilis & . & 168 & 2 & 4 & 2 & -2 & & & 0 \\
\hline Calamagrostis canescens & 4 & 78 & 3 & $9 \sim$ & 5 & 4 & & & 7 \\
\hline Caltha palustris & 4 & 245 & 2 & $8=$ & 5 & 4 & & & 6 \\
\hline Calystegia sepium & 3 & 168 & 2 & 6 & 4 & 2 & & & 4 \\
\hline Campanula patula & 2 & 246 & 2 & 5 & 3 & 0 & & & 2 \\
\hline Capsella bursa-pastoris & 1 & 262 & 1 & $\mathrm{x}$ & 3 & 0 & & & 1 \\
\hline Cardamine amara & 4 & 130 & 2 & $9=$ & 5 & 4 & & & 6 \\
\hline Cardamine pratensis & 3 & 230 & 2 & 7 & 4 & 2 & & & 4 \\
\hline Carduus crispus & . & 159 & 2 & 5 & 4 & 2 & & & 4 \\
\hline Carex acutiformis & 4 & 158 & 2 & $9 \sim$ & 5 & 4 & & & 6 \\
\hline Carex canescens & 4 & 86 & 3 & 9 & 5 & 4 & & & 7 \\
\hline Carex echinata & 4 & 118 & 3 & . & 5 & 4 & & & 7 \\
\hline Carex flava & 8 & 75 & 3 & 8 & 5 & 4 & & & 7 \\
\hline Carex gracilis & 4 & 108 & 3 & $9=$ & 5 & 4 & & & 7 \\
\hline Carex hirta & 1 & 241 & 2 & $6 \sim$ & $2-4$ & 0 & & & 2 \\
\hline Carex nigra & 4 & 199 & 2 & $8 \sim$ & $4-5$ & 3 & & & 5 \\
\hline Carex ovalis & 1 & 181 & 2 & $7 \sim$ & $3-4$ & 1 & & & 3 \\
\hline Carex panicea & 3 & 117 & 3 & $7 \sim$ & 4 & 2 & & & 5 \\
\hline Carex paniculata & 4 & 81 & 3 & $9=$ & 5 & 4 & & & 7 \\
\hline Carex pseudocyperus & 7 & 81 & 3 & 10 & 5 & 4 & & & 7 \\
\hline Carex riparia & 4 & 97 & 3 & $9=$ & 5 & 4 & & & 7 \\
\hline
\end{tabular}




\begin{tabular}{|c|c|c|c|c|c|c|c|c|c|}
\hline 1 & 2 & 3 & 4 & 5 & 6 & 7 & 8 & 9 & 10 \\
\hline Carex rostrata & 4 & 114 & 3 & 10 & 5 & 4 & & & 7 \\
\hline Carex spicata & . & 63 & 4 & 5 & $3-2$ & -0.5 & & & 3.5 \\
\hline Carex sylvatica & . & 141 & 2 & 5 & $3-4$ & 1 & & & 3 \\
\hline Carex vesicaria & 4 & 82 & 3 & $9=$ & 5 & 4 & & & 7 \\
\hline Carex vulpina & 4 & 109 & 3 & $9 \sim$ & 5 & 4 & & & 7 \\
\hline Centaurea jacea & 1 & 231 & 2 & $\mathrm{x}$ & 3 & 0 & & & 2 \\
\hline Centaurium erythraea & . & 115 & 3 & 5 & $2-3$ & -1 & & & 2 \\
\hline Cerastium arvense & 1 & 255 & 2 & 4 & 2 & -2 & & & 0 \\
\hline Cerastium holosteoides & 1 & 259 & 1 & 5 & $3-4$ & 1 & & & 2 \\
\hline Chaerophyllum temulum & . & 153 & 2 & 5 & 4 & 2 & & & 4 \\
\hline Chelidonium majus & . & 260 & 1 & 5 & 3 & 0 & & & 1 \\
\hline Chrysanthemum leucanthemum & 2 & 242 & 2 & 4 & 3 & 0 & & & 2 \\
\hline Circaea lutetiana & . & 164 & 2 & 6 & 4 & 2 & & & 4 \\
\hline Cirsium arvense & 1 & 262 & 1 & $\mathrm{x}$ & $2-3$ & -1 & & & 0 \\
\hline Cirsium oleraceum & 3 & 220 & 2 & 7 & $4-5$ & 3 & & & 5 \\
\hline Cirsium palustre & 3 & 231 & 2 & $8 \sim$ & 4 & 2 & & & 4 \\
\hline Cirsium vulgare & 1 & 260 & 1 & 5 & 3 & 0 & & & 1 \\
\hline Conyza canadensis & . & 257 & 1 & 4 & $2-3$ & -1 & & -4 & -4 \\
\hline Cornus sanguinea & . & 207 & 2 & $\mathrm{x}$ & $2-4$ & 0 & & & 2 \\
\hline Crataegus monogyna & . & 170 & 2 & 4 & $3-4$ & 1 & & & 3 \\
\hline Crepis biennis & 1 & 133 & 2 & 5 & 3 & 0 & & & 2 \\
\hline Cuscuta europaea & . & 54 & 4 & 7 & 4 & 2 & & & 6 \\
\hline Dactylis glomerata & 2 & 263 & 1 & 5 & 3 & 0 & & & 1 \\
\hline Daucus carota & 2 & 261 & 1 & 4 & 3 & 0 & & & 1 \\
\hline Deschampsia caespitosa & 3 & 259 & 1 & $7 \sim$ & 4 & 2 & & & 3 \\
\hline Deschampsia flexuosa & 1 & 225 & 2 & $\mathrm{x}$ & 3 & 0 & & & 2 \\
\hline Dianthus superbus & 8 & 82 & 3 & $8 \sim$ & 4 & 2 & 3 & & 8 \\
\hline Dryopteris filix-mas & 2 & 240 & 2 & 5 & $3-4$ & 1 & & & 3 \\
\hline Eleocharis palustris & 4 & 141 & 2 & $10 \sim$ & 5 & 4 & & & 6 \\
\hline Elymus caninus & 1 & 75 & 3 & 6 & 4 & 2 & & & 5 \\
\hline Elymus repens & 1 & 248 & 2 & $5 \sim$ & 3 & 0 & & & 2 \\
\hline Epilobium hirsutum & 3 & 184 & 2 & $8=$ & 5 & 4 & & & 6 \\
\hline Epilobium palustre & 4 & 143 & 2 & 9 & 5 & 4 & & & 6 \\
\hline Epilobium parviflorum & 4 & 77 & 3 & $9=$ & $4-5$ & 3 & & & 6 \\
\hline Equisetum arvense & 1 & 262 & 1 & $6 \sim$ & $3-4$ & 1 & & & 2 \\
\hline Equisetum fluviatile & 4 & 177 & 2 & 10 & 5 & 4 & & & 6 \\
\hline Equisetum palustre & 3 & 185 & 2 & 7 & 4 & 2 & & & 4 \\
\hline Equisetum pratense & 1 & 62 & 4 & 6 & 4 & 2 & & & 6 \\
\hline Eriophorum angustifolium & 4 & 75 & 3 & $9=$ & 5 & 4 & & & 7 \\
\hline Eupatorium cannabinum & 2 & 192 & 2 & 7 & 4 & 2 & & & 4 \\
\hline Euphorbia cyparissias & 1 & 249 & 2 & 3 & 2 & -2 & & & 0 \\
\hline Fallopia convolvulus & . & 251 & 2 & $\mathrm{x}$ & 3 & 0 & & -4 & -2 \\
\hline Festuca gigantea & . & 155 & 2 & 7 & 4 & 2 & & & 4 \\
\hline Festuca pratensis & 2 & 216 & 2 & 6 & 3 & 0 & & & 2 \\
\hline Festuca rubra & 2 & 219 & 2 & $\mathrm{x}$ & $2-4$ & 0 & & & 2 \\
\hline Filipendula ulmaria & 3 & 237 & 2 & 8 & $4-5$ & 3 & & & 5 \\
\hline Fraxinus excelsior & 2 & 250 & 2 & $\mathrm{x}$ & $4-3$ & 1.5 & & & 3.5 \\
\hline Galeobdolon luteum & . & 155 & 2 & 5 & 3 & 0 & & & 2 \\
\hline Galeopsis pubescens & . & 192 & 2 & 4 & $3-4$ & 1 & & & 3 \\
\hline Galeopsis tetrahit & 1 & 180 & 2 & 5 & $3-4$ & 1 & & & 3 \\
\hline Galium aparine & 1 & 247 & 2 & $\mathrm{x}$ & $4-3$ & 1.5 & & & 3.5 \\
\hline Galium mollugo & 2 & 248 & 2 & 5 & 3 & 0 & & & 2 \\
\hline Galium palustre & 4 & 223 & 2 & $9=$ & $4-5$ & 3 & & & 5 \\
\hline Galium uliginosum & 3 & 127 & 3 & $8 \sim$ & 4 & 2 & & & 5 \\
\hline Geranium palustre & 2 & 151 & 2 & $7 \sim$ & 5 & 3.5 & & & 5.5 \\
\hline Geranium pratense & 2 & 188 & 2 & 5 & 3 & 0 & & & 2 \\
\hline Geranium robertianum & 2 & 210 & 2 & $\mathrm{x}$ & 3 & 0 & & & 2 \\
\hline
\end{tabular}




\begin{tabular}{|c|c|c|c|c|c|c|c|c|c|}
\hline 1 & 2 & 3 & 4 & 5 & 6 & 7 & 8 & 9 & 10 \\
\hline Geum rivale & 3 & 176 & 2 & $8=$ & 4 & 2 & & & 4 \\
\hline Geum urbanum & . & 219 & 2 & 5 & $3-4$ & 1 & & & 3 \\
\hline Glechoma hederacea & 2 & 259 & 1 & 6 & $3-4$ & 1 & & & 2 \\
\hline Glyceria fluitans & 4 & 161 & 2 & $9=$ & $6-5$ & 6 & & & 8 \\
\hline Glyceria maxima & 4 & 206 & 2 & $10 \sim$ & 6 & 6 & & & 8 \\
\hline Gnaphalium uliginosum & . & 221 & 2 & 7 & 4 & 2 & & & 4 \\
\hline Helianthus tuberosus & . & 53 & 4 & 6 & $3-4$ & 1 & & -4 & 1 \\
\hline Holcus lanatus & 2 & 236 & 2 & 6 & 4 & 2 & & & 4 \\
\hline Hottonia palustris & 8 & 77 & 3 & 11 & 6 & 6 & & & 9 \\
\hline Humulus lupulus & 2 & 247 & 2 & $8=$ & $4-5$ & 3 & & & 5 \\
\hline Hydrocotyle vulgaris & 8 & 121 & 3 & $9 \sim$ & 4 & 3.5 & & & 6.5 \\
\hline Hypericum perforatum & 1 & 262 & 1 & 4 & $2-3$ & -1 & & & 0 \\
\hline Hypericum tetrapterum & 3 & 73 & 3 & $8=$ & 4 & 2 & & & 5 \\
\hline Hypochoeris radicata & . & 224 & 2 & 5 & 3 & 0 & & & 2 \\
\hline Impatiens noli-tangere & 2 & 177 & 2 & 7 & 4 & 2 & & & 4 \\
\hline Impatiens parviflora & 1 & 218 & 2 & 5 & 3 & 0 & & -8 & -6 \\
\hline Inula britannica & 2 & 174 & 2 & $7=$ & 4 & 2 & & & 4 \\
\hline Iris pseudacorus & 4 & 236 & 2 & 10 & 5 & 4 & & & 6 \\
\hline Isolepis setacea & . & 45 & 4 & 8 & 5 & 4 & 2 & & 10 \\
\hline Juncus articulatus & 4 & 184 & 2 & $8 \sim$ & $4-5$ & 3 & & & 5 \\
\hline Juncus bulbosus & . & 55 & 4 & 10 & $4-6$ & 4 & 2 & & 10 \\
\hline Juncus conglomeratus & 3 & 175 & 2 & $7 \sim$ & $4-5$ & 3 & & & 5 \\
\hline Juncus effusus & 3 & 253 & 2 & $7 \sim$ & $4-5$ & 3 & & & 5 \\
\hline Juncus tenuis & . & 156 & 2 & 6 & 4 & 2 & & & 4 \\
\hline Lamium maculatum & . & 181 & 2 & 6 & 4 & 2 & & & 4 \\
\hline Lathyrus palustris & 8 & 45 & 4 & $8=$ & $4-5$ & 3 & 1 & & 8 \\
\hline Lathyrus pratensis & 6 & 243 & 2 & 6 & $3-4$ & 1 & & & 3 \\
\hline Lemna minor & 4 & 234 & 2 & 11 & 6 & 6 & & & 8 \\
\hline Linaria vulgaris & 1 & 254 & 2 & 3 & $2-3$ & -1 & & & 1 \\
\hline Lotus corniculatus & 1 & 242 & 2 & 4 & $3-4$ & 1 & & & 3 \\
\hline Lotus uliginosus & 3 & 220 & 2 & $8 \sim$ & $4-5$ & 3 & & & 5 \\
\hline Luzula multiflora & 1 & 138 & 2 & $6 \sim$ & 3 & 0 & & & 2 \\
\hline Lychnis flos-cuculi & 3 & 253 & 2 & $6 \sim$ & 4 & 2 & & & 4 \\
\hline Lycopus europaeus & 4 & 232 & 2 & $9=$ & 5 & 4 & & & 6 \\
\hline Lysimachia nummularia & 3 & 243 & 2 & 6 & 4 & 2 & & & 4 \\
\hline Lysimachia thyrsiflora & 8 & 59 & 4 & $9=$ & 5 & 4 & & & 8 \\
\hline Lysimachia vulgaris & 4 & 249 & 2 & $8 \sim$ & $4-5$ & 3 & & & 5 \\
\hline Lythrum salicaria & 3 & 236 & 2 & $8=$ & $4-5$ & 3 & & & 5 \\
\hline Matricaria maritima subsp. inodora & . & 229 & 2 & $\mathrm{x}$ & 3 & 0 & & & 2 \\
\hline Medicago lupulina & 1 & 232 & 2 & 4 & $2-3$ & -1 & & & 1 \\
\hline Melandrium album & 4 & 256 & 2 & 4 & 3 & 0 & & & 2 \\
\hline Mentha aquatica & 4 & 197 & 2 & $9=$ & 5 & 4 & & & 6 \\
\hline Mentha longifolia & . & 133 & 2 & $8 \sim$ & $4-5$ & 3 & & & 5 \\
\hline Millium effusum & 1 & 169 & 2 & 5 & $3-4$ & 1 & & & 3 \\
\hline Molinia caerulea & 3 & 149 & 2 & $7 \sim$ & $4-5$ & 3 & & & 5 \\
\hline Myosotis palustris & 3 & 232 & 2 & $8 \sim$ & $4-5$ & 3 & & & 5 \\
\hline Myosotis sylvatica & . & 64 & 4 & 6 & $3-4$ & 1 & & & 5 \\
\hline Myosoton aquaticum & . & 190 & 2 & $8=$ & $4-5$ & 3 & & & 5 \\
\hline Odontites serotina & 1 & 166 & 2 & 5 & $3-4$ & 1 & & & 3 \\
\hline Oxalis fontana & . & 237 & 2 & 5 & 3 & 0 & & -4 & -2 \\
\hline Papaver rhoeas & . & 236 & 2 & 5 & 3 & 0 & & & 2 \\
\hline Peucedanum palustre & 4 & 185 & 2 & $9=$ & 5 & 4 & & & 6 \\
\hline Phalaris arundinacea & 4 & 220 & 2 & $8=$ & 5 & 4 & & & 6 \\
\hline Phleum pratense & 2 & 233 & 2 & 5 & $2-3$ & -1 & & & 1 \\
\hline Phragmites australis & 4 & 248 & 2 & $10 \sim$ & $5-6$ & 5 & & & 7 \\
\hline Pimpinella major & 3 & 123 & 3 & 6 & 3 & 0 & & & 3 \\
\hline Pimpinella saxifraga & 2 & 251 & 2 & 3 & 2 & -2 & & & 0 \\
\hline
\end{tabular}




\begin{tabular}{|c|c|c|c|c|c|c|c|c|c|}
\hline 1 & 2 & 3 & 4 & 5 & 6 & 7 & 8 & 9 & 10 \\
\hline Plantago lanceolata & 2 & 263 & 1 & $\mathrm{x}$ & $2-4$ & 0 & & & 1 \\
\hline Plantago major & 1 & 263 & 1 & 5 & $3-4$ & 1 & & & 2 \\
\hline Plantago media & 1 & 195 & 2 & 4 & $2-3$ & -1 & & & 1 \\
\hline Poa nemoralis & 1 & 211 & 2 & 5 & $2-3$ & -1 & & & 1 \\
\hline Poa palustris & 4 & 192 & 2 & $9=$ & $4-5$ & 3 & & & 5 \\
\hline Poa pratensis & 2 & 255 & 2 & 5 & 3 & 0 & & & 2 \\
\hline Poa trivialis & 1 & 161 & 2 & 7 & 4 & 2 & & & 4 \\
\hline Polygonum amphibium & 4 & 249 & 2 & $11 / 8 \sim$ & 6 & 6 & & & 8 \\
\hline Polygonum bistorta & 3 & 223 & 2 & $7 \sim$ & 4 & 2 & & & 4 \\
\hline $\begin{array}{l}\text { Polygonum lapathifolium subsp. } \\
\text { lapathifolium }\end{array}$ & . & 206 & 2 & 7 & $3-4$ & 1 & & & 3 \\
\hline Polygonum persicaria & . & 226 & 2 & 3 & 3 & 0 & & & 2 \\
\hline Populus tremula & 1 & 261 & 1 & 5 & 3 & 0 & & & 1 \\
\hline Potentilla anserina & 3 & 252 & 2 & $6 \sim$ & $3-4$ & 1 & & & 3 \\
\hline Potentilla argentea & 1 & 246 & 2 & 2 & 2 & -2 & & & 0 \\
\hline Potentilla erecta & 1 & 213 & 2 & $\mathrm{x}$ & $3-4$ & 1 & & & 3 \\
\hline Potentilla reptans & . & 245 & 2 & 6 & $3-4$ & 1 & & & 3 \\
\hline Prunella vulgaris & 1 & 236 & 2 & $\mathrm{x}$ & $3-4$ & 1 & & & 3 \\
\hline Ranunculus acris & 3 & 262 & 1 & $\mathrm{x}$ & $3-4$ & 1 & & & 2 \\
\hline Ranunculus auricomus & 3 & 164 & 2 & 6 & 4 & 2 & & & 4 \\
\hline Ranunculus flammula & 4 & 204 & 2 & $9 \sim$ & $4-5$ & 3 & & & 5 \\
\hline Ranunculus lingua & 4 & 53 & 4 & 10 & $6-5$ & 5 & 1 & & 10 \\
\hline Ranunculus repens & 3 & 261 & 1 & $7 \sim$ & $4-3$ & 1.5 & & & 2.5 \\
\hline Ranunculus sceleratus & 3 & 167 & 2 & $9=$ & $4-5$ & 3 & & & 5 \\
\hline Ribes spicatum & . & 108 & 3 & $8=$ & 4 & 2 & & & 5 \\
\hline Rorippa palustris & . & 237 & 2 & $9 \sim$ & 4 & 2 & & & 4 \\
\hline Rubus caesius & . & 146 & 2 & $7=$ & $2-4$ & 0 & & & 2 \\
\hline Rubus idaeus & 1 & 247 & 2 & 5 & $3-4$ & 1 & & & 3 \\
\hline Rumex acetosa & 2 & 260 & 1 & $\mathrm{x}$ & $3-4$ & 1 & & & 2 \\
\hline Rumex crispus & 1 & 247 & 2 & 6 & $3-4$ & 1 & & & 3 \\
\hline Rumex hydrolapathum & 4 & 147 & 2 & 10 & $5-6$ & 5 & & & 7 \\
\hline Rumex obtusifolius & 3 & 195 & 2 & 6 & $3-4$ & 1 & & & 3 \\
\hline Salix cinerea & 4 & 229 & 2 & $9 \sim$ & $4-5$ & 3 & & & 5 \\
\hline Salix fragilis & 1 & 248 & 2 & $8=$ & 4 & 2 & & & 4 \\
\hline Sambucus nigra & 2 & 262 & 1 & 5 & $3-4$ & 1 & & & 2 \\
\hline Sanguisorba officinalis & 1 & 204 & 2 & $7 \sim$ & 4 & 2 & & & 4 \\
\hline Scirpus sylvaticus & 3 & 224 & 2 & 9 & $4-5$ & 3 & & & 5 \\
\hline Scrophularia nodosa & 1 & 239 & 2 & 6 & 3 & 0 & & & 2 \\
\hline Scrophularia umbrosa & 1 & 91 & 3 & 10 & $4-5$ & 3 & & & 6 \\
\hline Scutellaria hastifolia & . & 48 & 4 & $8=$ & 4 & 2 & 4 & & 10 \\
\hline Senecio congestus & 8 & 40 & 4 & . & 5 & 4 & 3 & & 11 \\
\hline Senecio jacobaea & . & 235 & 2 & $4 \sim$ & $2-3$ & -1 & & & 1 \\
\hline Solanum dulcamara & 4 & 205 & 2 & $8 \sim$ & $5-4$ & 3.5 & & & 5.5 \\
\hline Solidago gigantea & . & 120 & 3 & 6 & $3-4$ & 1 & & -8 & -4 \\
\hline Sonchus asper & . & 186 & 2 & 6 & 3 & 0 & & & 2 \\
\hline Sparganium erectum & 4 & 143 & 2 & 10 & 6 & 6 & & & 8 \\
\hline Spiraea tomentosa & . & near 10 & 4 & . & . & 2 & & -8 & -2 \\
\hline Spirodela polyrhiza & 4 & 57 & 4 & 11 & 6 & 6 & & & 10 \\
\hline Stachys palustris & 4 & 215 & 2 & $7 \sim$ & $4-5$ & 3 & & & 5 \\
\hline Stellaria graminea & 1 & 221 & 2 & 4 & 3 & 0 & & & 2 \\
\hline Stellaria palustris & 4 & 73 & 3 & $8 \sim$ & $4-5$ & 3 & & & 6 \\
\hline Succisa pratensis & 3 & 140 & 2 & $7 \sim$ & 4 & 2 & & & 4 \\
\hline Symphytum officinale & 4 & 223 & 2 & 8 & $4-5$ & 3 & & & 5 \\
\hline Tanacetum vulgare & . & 247 & 2 & 5 & $3-4$ & 1 & & & 3 \\
\hline Taraxacum officinale & 3 & 263 & 1 & 5 & 3 & 0 & & & 1 \\
\hline Thalictrum flavum & 7 & 56 & 4 & $8 \sim$ & $4-5$ & 3 & 1 & & 8 \\
\hline Thelypteris palustris & 4 & 98 & 3 & 8 & $4-5$ & 3 & & & 6 \\
\hline
\end{tabular}




\begin{tabular}{|c|c|c|c|c|c|c|c|c|c|}
\hline 1 & 2 & 3 & 4 & 5 & 6 & 7 & 8 & 9 & 10 \\
\hline Tragopogon dubius & . & 29 & 5 & 4 & $2-3$ & -1 & & & 4 \\
\hline Tragopogon pratensis & 1 & 117 & 3 & 4 & 3 & 0 & & & 3 \\
\hline Trifolium hybridum & 3 & 170 & 2 & 6 & 4 & 2 & & & 4 \\
\hline Trifolium pratense & 2 & 259 & 1 & $\mathrm{x}$ & 3 & 0 & & & 1 \\
\hline Trifolium repens & 2 & 263 & 1 & $\mathrm{x}$ & $3-4$ & 1 & & & 2 \\
\hline Typha angustifolia & 4 & 119 & 3 & 10 & $6-5$ & 5 & & & 8 \\
\hline Typha latifolia & 4 & 218 & 2 & 10 & $6-5$ & 5 & & & 7 \\
\hline Urtica dioica & 2 & 263 & 1 & 6 & $3-4$ & 1 & & & 2 \\
\hline Utricularia vulgaris & 4 & 57 & 4 & 12 & 6 & 6 & & & 10 \\
\hline Veronica beccabunga & 3 & 173 & 2 & 10 & $5-6$ & 5 & & & 7 \\
\hline Veronica chamaedrys & 2 & 262 & 1 & 4 & 3 & 0 & & & 1 \\
\hline Veronica officinalis & 1 & 207 & 2 & 4 & 3 & 0 & & & 2 \\
\hline Veronica scutellata & 4 & 99 & 3 & $9=$ & $4-5$ & 3 & & & 6 \\
\hline Viburnum opulus & 5 & 216 & 2 & $\mathrm{x}$ & $3-4$ & 1 & & & 3 \\
\hline Vicia cracca & 2 & 210 & 2 & 5 & 3 & 0 & & & 2 \\
\hline Vicia hirsuta & . & 224 & 2 & $\mathrm{x}$ & 3 & 0 & & & 2 \\
\hline Vicia sepium & . & 161 & 2 & 5 & 3 & 0 & & & 2 \\
\hline Vicia tetrasperma & . & 223 & 2 & $5 \sim$ & 3 & 0 & & & 2 \\
\hline Viola palustris & 4 & 116 & 3 & 9 & $4-5$ & 3 & & & 6 \\
\hline Viola tricolor & 1 & 190 & 2 & $\mathrm{x}$ & 3 & 0 & & & 2 \\
\hline
\end{tabular}

Notes for column 5 (F (ElLENBERg 1979)): $\mathrm{x}$ - a wide amplitude (lack of indicatory character), - the indicator of variable moisture conditions, $=-$ the indicator of occurrence of periodic floodings.

"holes" e.g. between values from 1.8 to 1.9 and for classes II and III). The class boundaries which were set are presented in Table 1.

\section{EXAMINED OBJECTS AT THE BACKGROUND OF PHYSICAL-GEOGRAPHICAL AND GEOBOTANICAL REGIONALIZATION}

According to the physiographic division of Poland, eight objects were subject to the analysis (Fig. 1) which are situated in the scope of mesoregions: Pradolina Głogowska (318.32; Kotla, Głogówko), Bory Dolnośląskie (the Dolnośląskie Forest) (317.74; Parowa, Bronowiec), the boundary between Pradolina Wrocławska (318.52) and the Wrocław Plain (318.53; Przedmoście), the Wrocław Plain (318.53; Miękinia), Wysoczyzna Kaliska (318.12; Milicz). According to the geobotanical division of Poland, they are found in such districts as: Lubuskie (Kotla, Głogówko) and Baryckie (Milicz), the Śląsko-Łużycka Lowland with the Sub district of Bory Dolnośląskie (Parowa, Bronowiec) as well as the Śląska Lowland with the Sub district of the Chojnowsko-Legnicko-Wrocławska Plain (Przedmoście, Miękinia) (Szafer 1972, KONDRACKI 1994).

The objects which were earlier managed are mostly abandoned at present. In such areas, specific flora comes into existence representing various succession stages. There are also formed untypical stages of common flora communities (compare: WotejKO 2000).

\section{RESULTS OF EXAMINATIONS AND DISCUSSION}

\section{FLORA AND VEGETATION OF THE EXAMINED OBJECTS}

In the swamp-originating meadows of Lower Silesia, there were altogether recorded 235 species of vascular plants and 26 types of phytocoenoses (PoDLASKA 2009). In the examined meadows there were distinguished eight types of plant associations and one plant community in the rank of a plant association [after: Matuszkiewicz 2006]. They are communities included in the class of Phragmitetea R.Tx. et Prsg 1942: Phragmitetum australis (Gams 1927) Schmale 1939, Cicuto-Caricetum pseudocyperi Boer et Siss. in Boer 1942, Caricetum acutiformis Sauer 1937, Caricetum rostratae Rübel 1912, Caricetum gracilis (Graebn. et Hueck 1931) R.Tx. 1937, Phalaridetum arundinaceae (Koch 1926 n.n.) Libb. 1931, and reckoned among the class of Molinio-Arrhenatheretea R.Tx. 1937: Scirpetum sylvatici Ralski 1931, Alopecuretum pratensis (Regel 1925) Steffen 1931, as well as the community of Deschampsia caespitosa = Deschampsietum caespitosae (Horvatić 1930) (PoDLASKA 2009).

The remaining phytocoenoses are incomplete (impoverished) communities composed mainly of segetal, ruderal and invasive species. Even if they are composed of species typical for wetland systems or swamp-originating meadows, their species composition was greatly altered. There were distinguished: Alnus glutinosa, Lysimachia vulgaris, Juncus conglomeratus, Carex nigra and Juncus conglomeratus, Carex nigra, Carex panicea, Holcus lanatus, Elymus caninus, Bromus 


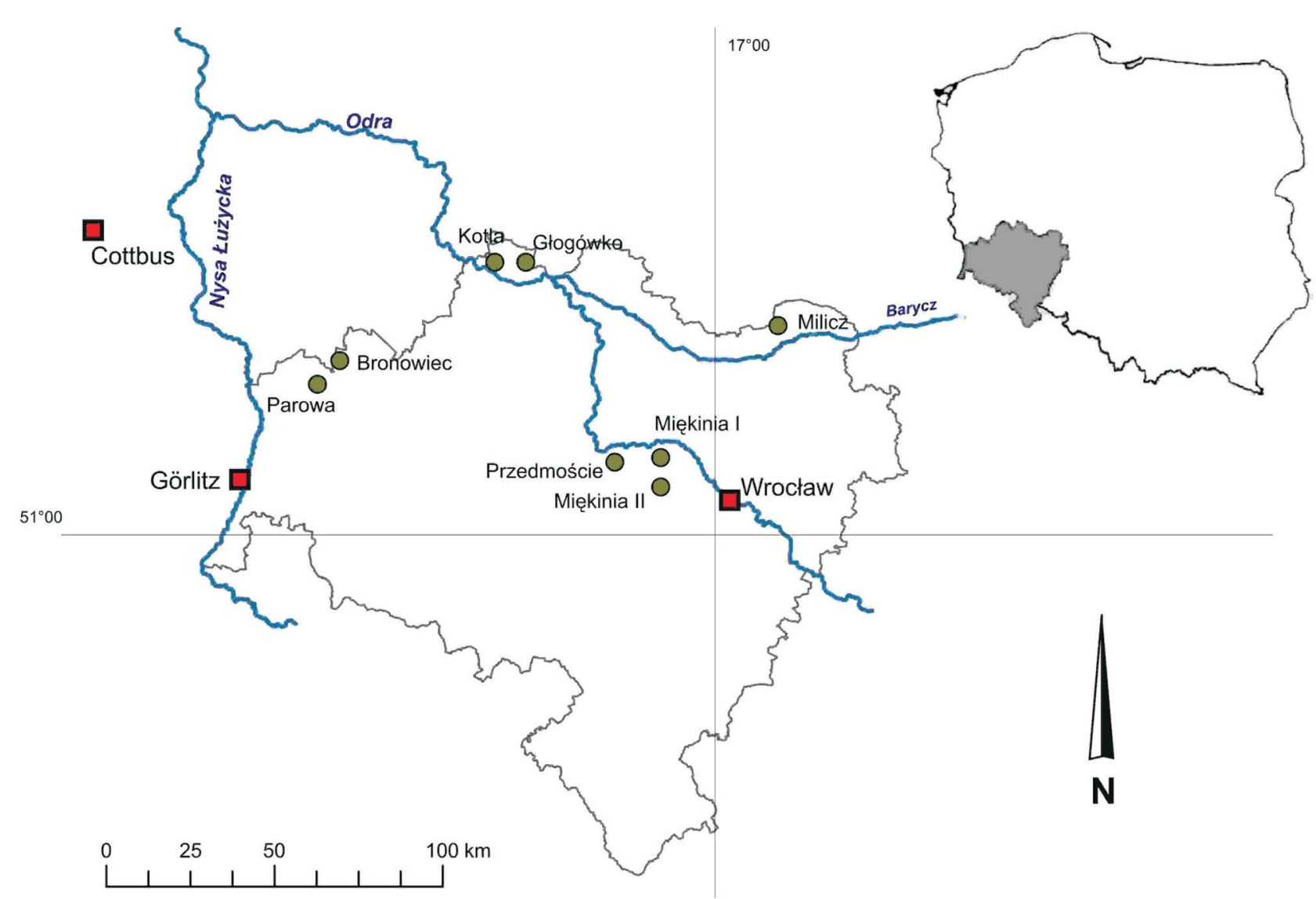

Fig. 1. Distribution of the examined objects

inermis, Calamagrostis canescens, Urtica dioica, Galium aparine, Cirsium arvense, Solidago gigantea, Solidago gigantea and Impatiens parviflora, Spiraea tomentosa.

\section{NATURAL VALUES OF PHYTOCOENOSES}

The evaluation was conducted for all the found phytocoenoses according to the OśwIT (2000) method and to the author's method. The results of the entire evaluation were presented in Table 3 . With the purpose of indicating differences in the evaluations conducted by use of both methods, two types of phytocoenoses were selected - the well-formed communities Phragmitetum australis and the community of invasive species Spiraea tomentosa (Tables 4 and 5).

Field observations of phytoceonoses Phragmitetum australis (Table 4) did not indicate a significant variation of reeds in respective objects, however nowhere their patches were preserved optimally (significant fragmentation, suboptimal habitat conditions, ecologically alien species or geographically penetrating into the patches etc). According to the OśwIT (2000) method, cane reeds fall into classes from VI to VIII (values from moderately high to significantly high), whereas the highest values were acquired by reeds in the objects of Parowa and Miekinia I, the lowest by reeds in Bronowiec (in spite of their values comparable with the former).
According to the author's method, the phytocenoses Phragmitetum australis exhibited lower natural values in the range of classes from IV to VI (values from moderate to moderately high), which more clearly reflected their real value. The reeds of Parowa and Miękinia I acquired the highest values, the lowest values were obtained by the reeds of Głogówko.

The brushwood Spiraea tomentosa found in Parowa is composed of an invasive species, which classifies such objects as naturally invaluable. Even the Oświt (2000) elaboration did not include the valorization value for that kind of species. Among accompanying species there prevail taxa of higher valorization values, but their amount in the patches of spirea is marginal. According to the Oświt method, the presence of these species allows areas in which Spiraea tomentosa grows to be reckoned among class III (low values), which however does not reflect the real state of things. According to the author's method, these areas are reckoned among class II (moderately low values).

\section{NATURAL VALUES OF RESPECTIVE EXAMINED OBJECTS}

The variability of natural values of the whole examined objects was also put to analysis due to the large floristical variation of respective swamp-originating meadows (Table 3 ). 


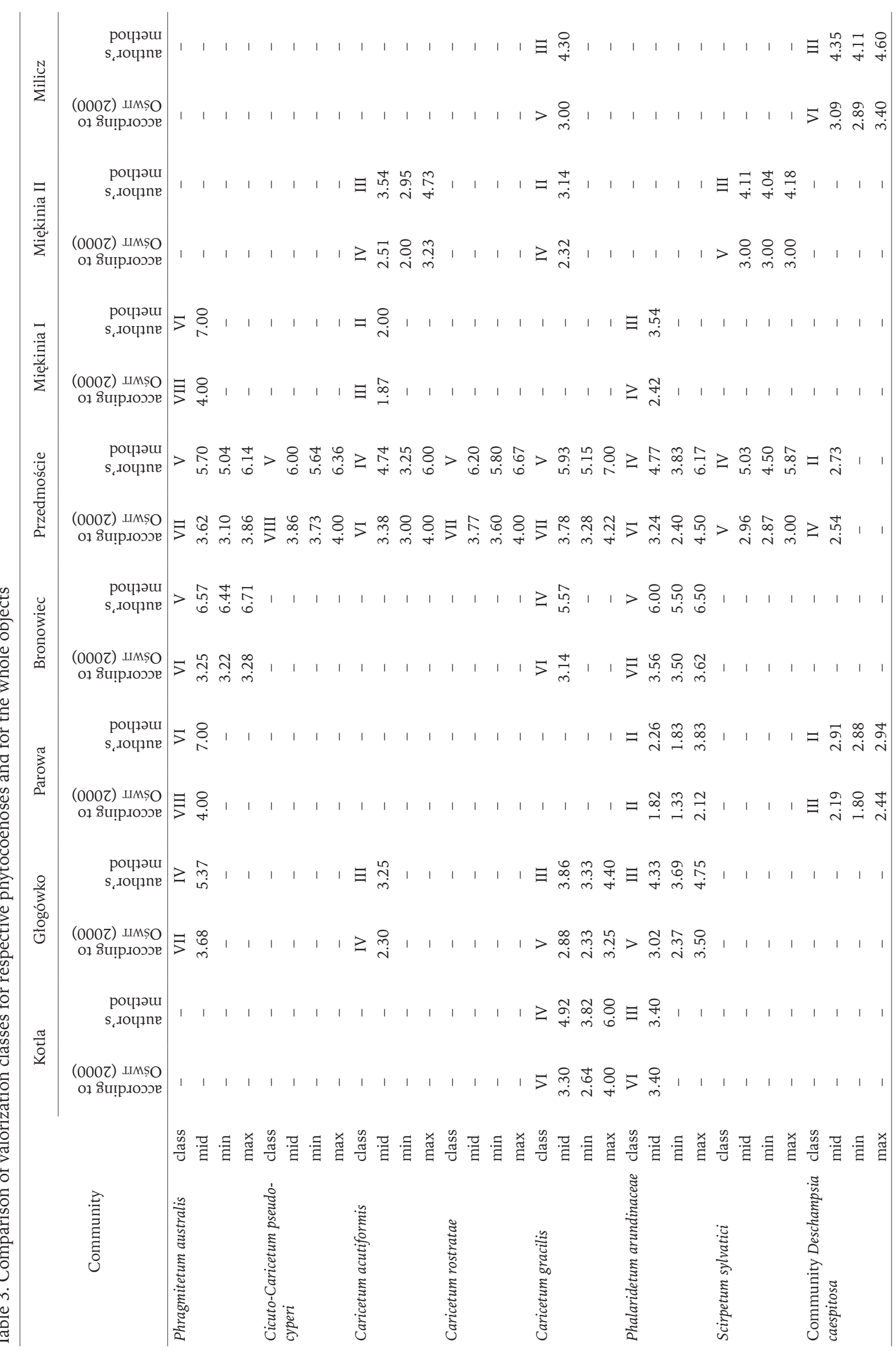




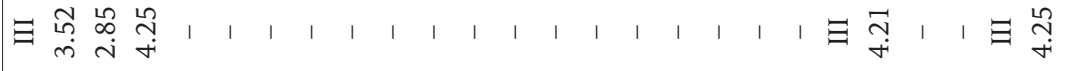

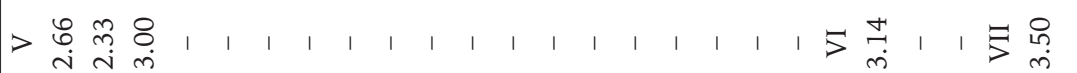

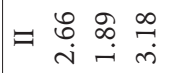

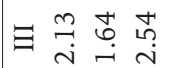

$$
\begin{aligned}
& \text { - }
\end{aligned}
$$

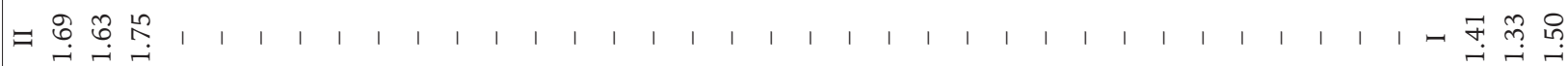

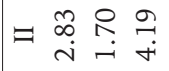

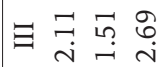

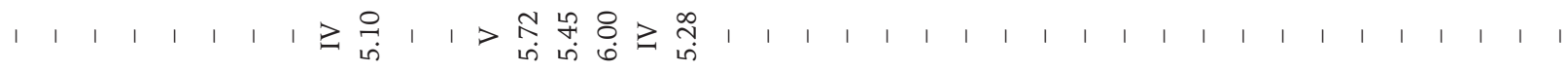

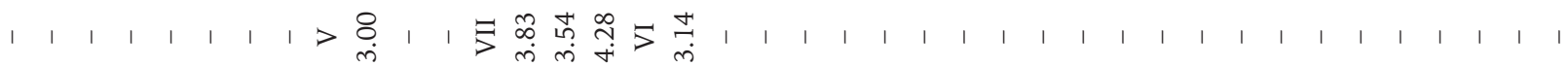

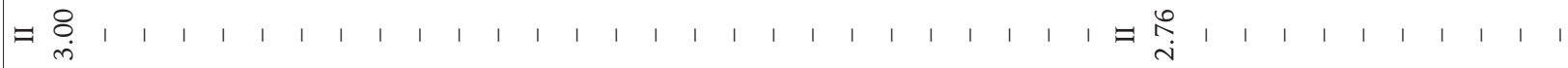

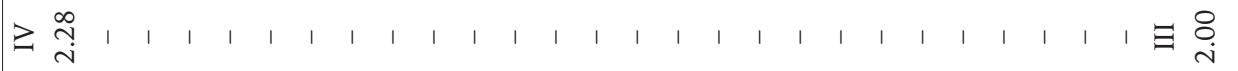

$$
\begin{aligned}
& \text { 龍 }
\end{aligned}
$$

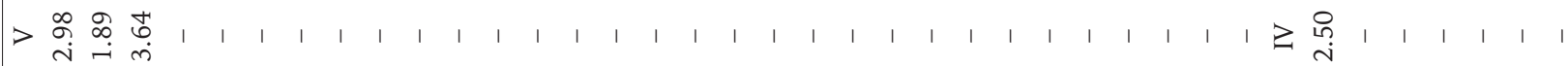

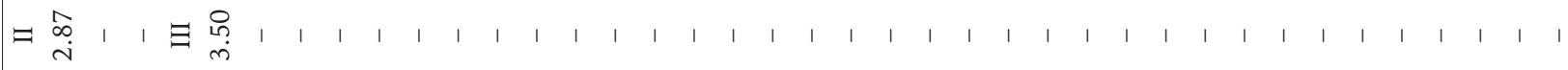

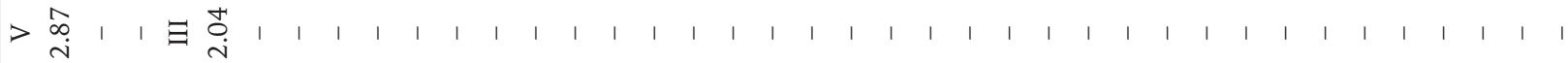

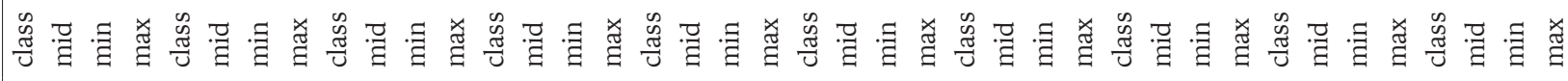

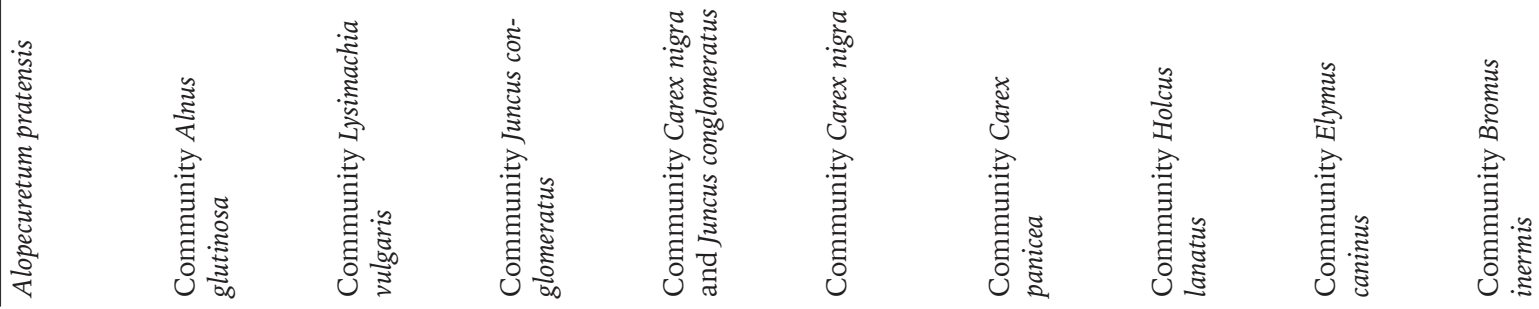




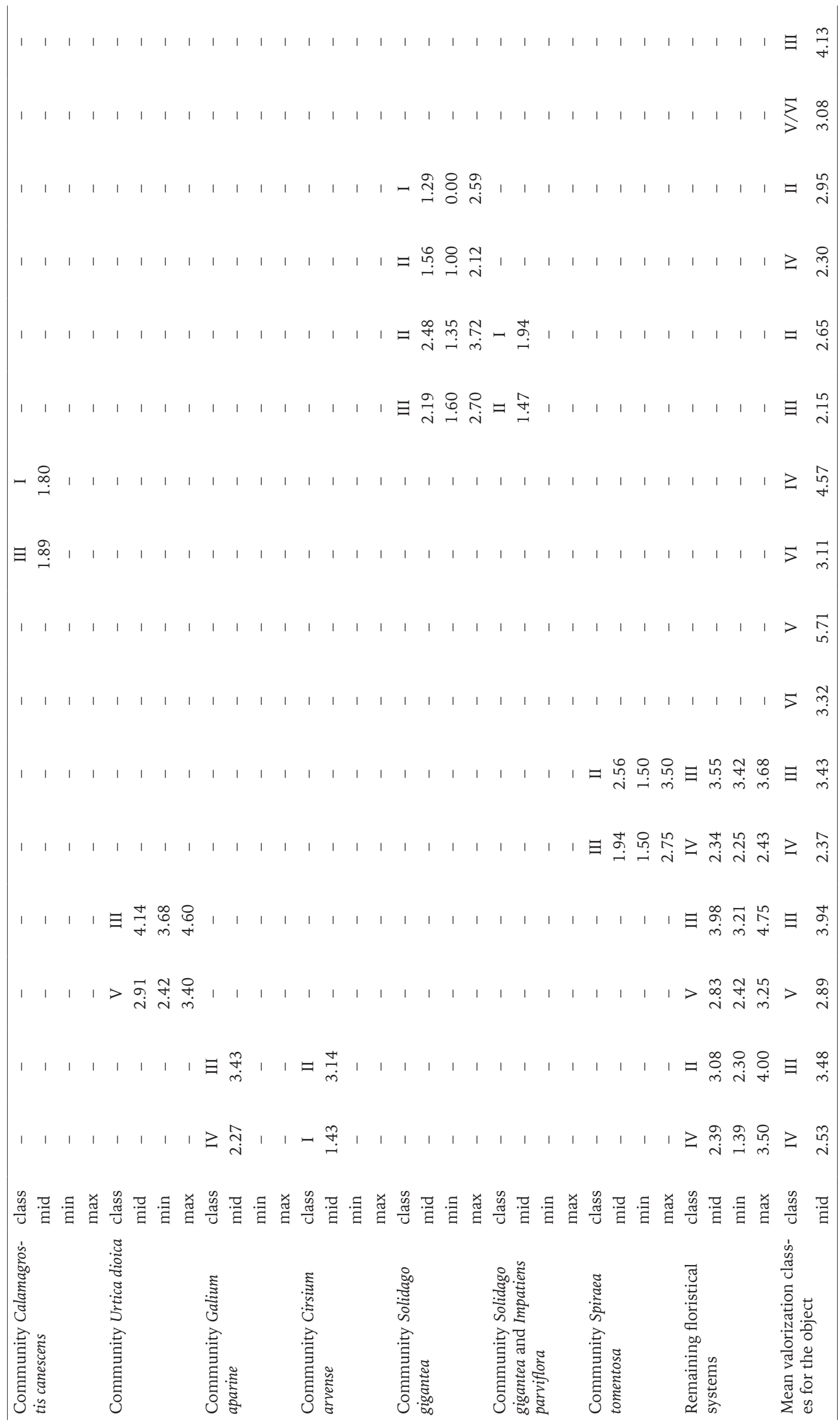


Tabela 4. The evaluation of natural values for the association Phragmitetum australis in the examined objects

\begin{tabular}{|c|c|c|c|c|c|c|c|c|c|c|c|c|c|}
\hline \multirow{2}{*}{ Species } & \multirow{2}{*}{$\mathrm{LW}_{\mathrm{O}}$} & \multirow{2}{*}{$\mathrm{LW}_{\mathrm{w}}$} & \multirow{2}{*}{$\begin{array}{c}\text { Gł. } \\
4\end{array}$} & \multirow{2}{*}{$\begin{array}{c}\text { Par. } \\
1\end{array}$} & \multicolumn{2}{|c|}{ Bro. } & \multicolumn{6}{|c|}{ Prz. } & \multirow{2}{*}{$\begin{array}{c}\text { M I } \\
2\end{array}$} \\
\hline & & & & & 4 & 5 & 6 & 7 & 8 & 9 & 10 & 11 & \\
\hline Acorus calamus & 4 & 3 & + & . & . & . & . & . & . & . & . & . & . \\
\hline Agrostis stolonifera & 4 & 4 & + & . & . & . & . & . & . & . & . & . & . \\
\hline Alisma plantago-aquatica & 4 & 7 & + & . & . & . & . & . & . & . & . & . & . \\
\hline Alopecurus geniculatus & 3 & 6 & + & . & . & . & . & . & . & . & . & . & . \\
\hline Bidens tripartita & - & 5 & . & . & + & + & . & . & . & . & . & . & . \\
\hline Calystegia sepium & 3 & 4 & . & . & . & . & . & . & . & + & + & . & . \\
\hline Carex acutiformis & 4 & 6 & . & . & . & . & . & . & + & + & + & + & . \\
\hline Carex gracilis & 4 & 7 & + & . & + & . & . & + & + & + & . & . & . \\
\hline Carex rostrata & 4 & 7 & + & . & . & . & . & . & . & . & . & . & . \\
\hline Carex vesicaria & 4 & 7 & . & . & . & . & . & . & + & . & . & . & . \\
\hline Epilobium palustre & 4 & 6 & . & . & . & + & . & . & . & . & . & . & . \\
\hline Epilobium parviflorum & 4 & 6 & + & . & . & . & + & . & . & . & + & . & . \\
\hline Equisetum palustre & 3 & 4 & . & . & . & . & + & . & . & . & . & . & . \\
\hline Galium aparine & 1 & 3.5 & . & . & . & . & . & . & . & + & . & . & . \\
\hline Galium palustre & 4 & 5 & + & . & . & . & . & . & . & . & + & + & . \\
\hline Galium uliginosum & 3 & 5 & . & . & + & + & . & . & . & . & . & . & . \\
\hline Glechoma hederacea & 2 & 2 & . & . & . & . & . & . & . & . & + & . & . \\
\hline Hottonia palustris & 8 & 9 & + & . & . & . & . & . & . & . & . & . & . \\
\hline Impatiens noli-tangere & 2 & 4 & . & . & . & . & . & + & . & . & . & . & . \\
\hline Iris pseudacorus & 4 & 6 & + & . & . & . & . & . & . & + & . & + & . \\
\hline Juncus conglomeratus & 3 & 5 & . & . & + & . & . & . & . & . & . & . & . \\
\hline Juncus effusus & 3 & 5 & + & . & + & . & . & . & . & . & . & . & . \\
\hline Lemna minor & 4 & 8 & . & . & + & + & + & . & . & . & . & . & . \\
\hline Lycopus europaeus & 4 & 6 & . & . & + & + & + & . & . & . & . & . & . \\
\hline Lysimachia vulgaris & 4 & 5 & . & . & . & . & . & . & . & + & + & + & . \\
\hline Lythrum salicaria & 3 & 5 & . & . & . & . & + & . & + & + & . & + & . \\
\hline Mentha aquatica & 4 & 6 & + & . & . & . & . & . & . & . & . & . & . \\
\hline Phalaris arundinacea & 4 & 6 & . & . & . & . & . & . & + & . & . & . & . \\
\hline Phragmites australis & 4 & 7 & + & + & + & + & + & + & + & + & + & + & + \\
\hline Polygonum persicaria & - & 2 & + & . & . & . & . & . & . & . & . & . & . \\
\hline Ranunculus repens & 3 & 2.5 & + & . & . & . & . & . & . & . & . & . & . \\
\hline Rumex crispus & 1 & 3 & + & . & . & . & . & . & . & . & . & . & . \\
\hline Scirpus sylvaticus & 3 & 5 & . & . & . & . & + & . & . & . & . & . & . \\
\hline Scutellaria hastifolia & - & 10 & . & . & . & . & . & . & . & + & . & . & . \\
\hline Solanum dulcamara & 4 & 5.5 & + & . & . & . & . & . & . & . & + & . & . \\
\hline Symphytum officinale & 4 & 5 & + & . & . & . & . & + & + & + & + & + & . \\
\hline Thalictrum flavum & 7 & 8 & . & . & . & . & . & . & . & . & + & . & . \\
\hline Urtica dioica & 2 & 2 & . & . & . & . & . & . & . & . & + & . & . \\
\hline Utricularia vulgaris & 4 & 10 & . & . & + & + & . & . & . & . & . & . & . \\
\hline Veronica scutellata & 4 & 6 & + & . & . & . & . & . & . & . & . & . & . \\
\hline \multicolumn{3}{|c|}{ Number of species in the picture } & 19 & 1 & 9 & 7 & 7 & 4 & 7 & 10 & 11 & 7 & 1 \\
\hline \multicolumn{3}{|c|}{$\Sigma$ of valorization points $[\mathrm{A}]$} & 102 & 7 & 58 & 47 & 41 & 23 & 43 & 58.5 & 55.5 & 39 & 7 \\
\hline \multicolumn{3}{|c|}{ Mean valorization value } & 5.37 & 7.00 & 6.44 & 6.71 & 5.86 & 5.75 & 6.14 & 5.85 & 5.04 & 5.57 & 7.00 \\
\hline \multicolumn{3}{|c|}{ Mean valorization value for the association } & 5.37 & 7.00 & \multicolumn{3}{|c|}{6.57} & \multicolumn{4}{|c|}{5.70} & & 7.00 \\
\hline Mean valorization class & & & IV & VI & & $\mathrm{V}$ & & & & & & $\mathrm{V}$ & VI \\
\hline \multicolumn{3}{|c|}{$\Sigma$ of valorization points $[\mathrm{O}]$} & 70 & 4 & 29 & 23 & 25 & 14 & 27 & 31 & 42 & 27 & 4 \\
\hline \multicolumn{3}{|c|}{ Mean valorization value } & 3.68 & 4.00 & 3.22 & 3.28 & 3.57 & 3.50 & 3.86 & 3.10 & 3.82 & 3.86 & 4.00 \\
\hline \multicolumn{3}{|c|}{ Mean valorization value or the association } & 3.68 & 4.00 & & & & & & & & & 4.00 \\
\hline Mean valorization class & & & VII & VIII & & VI & & & & & & VII & VIII \\
\hline
\end{tabular}

Notes: LW - valorization value according to OśwIT (2000); $\mathrm{LW}_{\mathrm{W}}$ - valorization value according to the author's method; Gł. - Głogówko, Par. - Parowa, Bro. - Bronowiec, Prz. - Przedmoście, M I - Miękinia I; [A] - the author’s method, [O] - the Oświt method.

According to the OśwIT (2000) classification the natural value of Kotla phytocoenoses is from moderate to moderately high (classes IV-VI prevail). Individually, there occur phytocoenoses of very low and low natural values (classes I and III). According to the author's method, there prevail phytocoenoses of averagely low and low values, and an individual phytocoenosis indicates moderate values (classes II-III 
Table 5. Evaluation of natural values for the community Spiraea tomentosa in the object of Parowa

\begin{tabular}{|c|c|c|c|c|c|c|}
\hline \multirow{2}{*}{ Species } & \multirow{2}{*}{$\mathrm{LW}_{\mathrm{O}}$} & \multirow{2}{*}{$\mathrm{LW}_{\mathrm{w}}$} & \multicolumn{4}{|c|}{ Community Spiraea tomentosa } \\
\hline & & & 10 & 11 & 12 & 13 \\
\hline Betula pendula & - & 1 & . & + & . & . \\
\hline Carex nigra & 4 & 5 & + & . & . & . \\
\hline Deschampsia caespitosa & 3 & 3 & + & . & . & . \\
\hline Deschampsia flexuosa & 1 & 2 & . & . & . & + \\
\hline Holcus lanatus & 2 & 4 & + & . & . & . \\
\hline Juncus conglomeratus & 3 & 5 & + & . & . & . \\
\hline Juncus effusus & 3 & 5 & . & + & + & . \\
\hline Lysimachia vulgaris & 4 & 5 & + & . & . & . \\
\hline Molinia caerulea & 3 & 5 & . & + & . & . \\
\hline Phalaris arundinacea & 4 & 6 & + & + & . & . \\
\hline Phragmites australis & 4 & 7 & . & . & . & + \\
\hline Poa pratensis & 2 & 2 & + & . & . & + \\
\hline Spiraea tomentosa & - & -2 & + & + & + & + \\
\hline Number of species in the picture & & & 8 & 5 & 2 & 4 \\
\hline$\Sigma$ of valorization points $[\mathrm{A}]$ & & & 28 & 15 & 3 & 9 \\
\hline Mean valorization value & & & 3.5 & 3.00 & 1.50 & 2.25 \\
\hline Mean valorization value for the community & & & \multicolumn{4}{|c|}{2.56} \\
\hline Mean valorization class & & & & & & \\
\hline$\Sigma$ of valorization points $[\mathrm{O}]$ & & & 22 & 10 & 3 & 6 \\
\hline Mean valorization value & & & 2.75 & 2.00 & 1.50 & 1.50 \\
\hline Mean valorization value for the community & & & & & & \\
\hline Mean valorization class & & & & & & \\
\hline
\end{tabular}

Notes: $\mathrm{LW}_{\mathrm{O}}$ - valorization value according to OśwIT (2000); $\mathrm{LW}_{\mathrm{W}}$ - valorization value according to the author's method; [A] - the author's method, [O] - the Oświt method.

and IV). According to the Oświt method, the mean valorization value for the whole object calculated on the basis of respective phytocoenoses falls into class IV (moderate values), but it falls into class III (low values) according to the author's method.

According to the OśwIT (2000) method, in Głogówko there prevail communities of moderate and averagely moderate values (classes IV-V), an individual phytocoenosis indicates high values (class VII). According to the author's method, there predominate communities of low values (class III), individually there occur communities of averagely low and high values (classes I and VII). According to the Oświt method, the mean valorization value for the whole object falls into class $\mathrm{V}$ (averagely moderate values), and according to the author's method it falls into class III (low values).

The presence of the invasive species Spiraea tomentosa diminishes the value of communities which occur in habitats of disturbed water relations and advanced peat mineralization in Parowa. According to the OśwIT (2000) classification, there predominate communities of averagely low to moderate values (classes II-IV), an individual phytocoenosis falls into class VIII (very high values). In the author's method there prevail phytocoenoses of averagely low and low values (classes II-III), an individual phytocoenosis falls into class VI (moderately high values). According to the Oświt method, the mean valorization value for the whole object falls into class IV (moderate val- ues) but into class III (low values) according to the author's method.

According to the Oświt (2000) method, the communities in Bronowiec obtain from averagely moderate to high values (classes V-VII). According to the author's method the natural values of these phytoceonoses are lower - they fall into classes IV-V (moderate to averagely moderate values). According to the Oświt method, the mean valorization values for the whole object fall into class VI (moderately high values), but into class $\mathrm{V}$ (averagely moderate values) according to the author's method. From the natural point of view, at present there occurs a very beneficial process of secondary swamping. As a result of water stagnation, such areas are invaded by phytocoenoses of wetland flora including peat forming and protected species - e.g. Utricularia vulgaris. As the reconstruction of the swampy habitat has occurred relatively recently, some plant communities undergo early stages of secondary succession. The values are decreased by the presence of the invasive species Spiraea tomentosa.

The complex of swamp-originating meadows of Przedmoście is strongly diversified. According to the Oświt (2000) method, as a result of high variability of moisture conditions of various parts of the site, in secondarily swamping habitats there occur very valuable, including peat forming, communities (classes VI-VIII; moderately high to very high values) and less valuable communities typical for wet and fresh swamp-originating meadows (classes IV-V; moder- 
ate to averagely moderate values), as well as strongly degenerated forms of these meadows (class III, low values). According to the author's method, there predominate communities of moderate to averagely moderate values (classes IV-V), individually there occur communities of very low to averagely low values (classes I-II). According to the Oświt method, the mean valorization value for the whole object falls into class VI (moderately high values), but into class IV (moderate values) according to the author's method. The value of meadow complexes is increased by the presence of protected species and so is the value for the whole object owing to the presence of beavers which beneficially transform the habitat.

In the object of Miękinia I the natural value of communities is low, as swamp-originating meadows have to a large degree been destroyed. According to the Oświt (2000) method, there predominate classes I-IV (very low to moderate values) and an individual phytocoenosis falls into class VIII (very high values). According to the author's method, there prevail classes I-III (very low to low values), individually there occurs class VI (moderately high values). According to the Oświt method, the mean valorization value for the whole object falls into class III (low values), but into class II (averagely low values) according to the author's values. From the natural point of view, the presence of beavers has a beneficial influence on it.

The natural values of the Miękinia II object are averagely low to averagely moderate (classes II-V) according to the Oświt (2000) method. The author's method indicates their even lower values - they fall into classes I-III (very low to low values). According to the Oświt method, the mean valorization value for the whole object falls into class IV (moderate values), but into class II (averagely low values) according to the author's method. Such an outcome results from an advanced degradation of its habitats due to their significant dryness and a massive occurrence of the invasive species Solidago gigantea and Impatiens parviflora. In spite of significant degradation of the habitats, in the examined objects there still occur species of protected plants typical for peatland systems: Centaurium erythraea, Dianthus superbus and Viburnum opulus.

In the object of Milicz there predominate communities typical for swamp-originating meadows - Deschampsia cespitosa and degenerated foxtail meadows, the actual natural value of which is insignificant. According to the Oświt (2000) method, the occurring phytocoenoses are included into classes V-VII (averagely moderate to high values), but according to the author's method all the phytocoenoses fall into class III (low values). According to the Oświt method, the mean valorization value for the whole object is included in class V/VI (averagely moderate to moderately high values; discontinuity of the scale is evident in the Oświt method), but it falls into class III (low values according to the author's method.

The analysis of natural values of the Lower Silesia swamp-originating meadows indicates that the natural value of both respective objects and their phytocoenoses is fairly diversified. It is so due to a different degree of formation of respective plants patches, and due to various processes which occurred in these meadows in the past and which are occurring at present. Evaluation of the natural value of such meadows by use of the OśwIT (2000) method produced an overestimated result. The values obtained in the valorization process by use of the author's method are closer to the actual state (recorded in the field) of respective phytocoenoses.

The marshy meadow valleys, in which low peatlands are situated, are included in the areas formed by variable fluvial processes, mainly periodic surface floodings. Such conditions gave rise to a wide variety of biotopes characterized by very high natural values (OśwIT 1996). The natural values of peatland objects, situated within the area of such valleys, are generally significant (URBAN \& GRZYWNA 2003) provided these are living peatlands. All the examined objects of Lower Silesia once underwent melioration and currently they represent a better or worse post-melioration state. As a result of these activities and due to the disappearance of peatland flora there developed substitute communities of grasslands. The natural value of such objects is variously evaluated - e.g. ZAŁUSKI \& KAMiŃSKA (1999) and SzTYBER (2004) state that grassland complexes in objects of that type do not generally indicate high natural values although, as many of them play a non-productive role, they hold a significant sway over hydrological, microclimatic, hydro sanitary and biocenotic conditions. In place of peat forming phytocoenoses there develop substitute meadow communities. The other authors (OlaczeK et al. 1990, KochanOWSKA 1997, BACIECZKO 1999, GrzelaK et al. 2002/2003, GrzelaK \& Bocian 2006 ) indicate that meadow communities, in various stages of secondary succession, are characterised by undeniable natural values. Abandoned grasslands, in spite of the lack of economic productivity, fulfill important ecological functions because they frequently serve as a shelter and refuge for various plants and animals (mainly entomo- and avifauna), they maintain bioversity of agricultural landscape, they constitute a diaspora bank for natural flora and they play a significant role in the life of a human being.

After the post-melioration development of marshy ecosystems there frequently come into existence communities of low natural values - phytocoenoses of moderate values survive solely in wet habitats as these create good conditions for maintaining marshy species. Habitats of a various degree of moisture conditions and intensity of use are characterised by different natural values. Dry habitats used intensively 
and extensively indicate very low natural values. Variably wet habitats used intensively are characterised by low natural values and those used extensively by averagely low values. Wet habitats used both intensively and extensively indicate moderate natural values (KRYSZAK et al. 2006).

In most cases the natural value of phytocoenoses of the examined swamp-originating meadows varies substantially from the value given for optimally formed phytocoenoses of low peatlands (OśwIT 2000). The examined objects, despite their far reaching transformations, are still featured by a significant natural value. At present, new and extremely varied systems are formed - from swampy to extremely dry. Areas which spontaneously undergo secondarily swamping are exceedingly valuable from the natural point of view, as they are invaded by species and plant communities similar to natural ones. The calculated values of valorization classes are often largely overestimated (in relation to the species composition of phytocoenoses), which in case of most phytocoenoses (in particular those including dominant invasive species) results from an increase in the class value caused by a marginal occurrence of species of a high valorization value. Hence, on evaluating the natural value of a given community it is necessary to take into account the floristical and phytosociological analysis, as only such a juxtaposition produces a more accurate image of an examined object.

\section{CONCLUSIONS}

1. The valorization of the Lower Silesia swamp-originating meadows was carried out by use of the Oświt method and the author's method. Either of these methods offered a different approach towards the natural valorization of the marshy land, in particular swamp-originating meadows.

2. On the basis of the author's method the valorization values were calculated for all the species of vascular flora which spontaneously occur in the examined meadows. In relation to the Oświt method, the valorization values for 27 species were decreased, for 125 they were increased and for 33 they remained unchanged. The valorization values were also determined for 50 species which were not included in the Oświt elaboration.

3. The comparison of the natural values evaluation carried out by use of the Oświt method and the author's method indicates that the author's method classified the phytocoenoses of swamp-originating meadows and their whole complexes more adequately in respect of the degree of their degradation. According to the author's method, the phytocoenoses in the researched areas obtained lower values than in the case of the Oświt method.

\section{REFERENCES}

BACIECZKO W. (1999): Roślinność wilgotnych łąk i ziołorośli w dolinie Płoni ostoją różnorodności florystycznej. Folia Universitatis Agriculturae Stetinensis 197, Agricultura 75: 11-18.

BADANIA złóż torfowych $\mathrm{w}$ województwie legnickim pod kątem ich znaczenia przyrodniczo-gospodarczego. Część XV. Rejon Głogówko. (1983 a). Typescript. KBiER, UP Wrocław.

BADANIA złóż torfowych w województwie legnickim pod kątem ich znaczenia przyrodniczo-gospodarczego. Część XVI. Rejon Chociemyśl. (1983b). Typescript. KBiER, UP Wrocław.

ElLENBERG H. (1979): Zeigerwerte der Gefäßpflanzen Mitteleuropas. Scripta Geobotanica 9.

GrYNIA M. (1980): Znaczenie zbiorowisk łąkowo-pastwiskowych w środowisku przyrodniczym niektórych regionów kraju. Roczniki Akademii Rolniczej w Poznaniu 118: 39-46.

Grzelak M., Bocian T. (2006): Zróżnicowanie geobotaniczne zbiorowisk seminaturalnych doliny Noteci Bystrej oraz ich rola w krajobrazie. Annales Universitatis Mariae Curie-Skłodowska, Sectio E, 61: 257-266.

Grzelak M., Kryszak A., Spychalski W. (2002/2003): Charakterystyka geobotaniczna zbiorowisk szuwarowych związku Phragmition w wybranych dolinach rzecznych Wielkopolski. Roczniki Akademii Rolniczej w Poznaniu 353, Rolnictwo 62: 15-23.

ILNICKI P., ed. (2002): Torf i torfowiska. Wyd. Akademii Rolniczej im. Augusta Cieszkowskiego w Poznaniu, Poznań.

Ilnicki P., Dardas J., Sikora K., Tadrowska A., TrzaSKOWSKA L., WoŹNIAK A. (2004): Zmiany sposobu użytkowania torfowisk Wielkopolski. Woda - Środowisko - Obszary Wiejskie 4, 1(10): 357-371.

JASNOWSKI M. (1972): Rozmiary i kierunki przekształceń szaty roślinnej torfowisk. Phytocoenosis 173: 193-209.

KalbarCZYK M. (1982): Stratygrafia, gleby i wartość gospodarcza torfowiska „Piaski” w rejonie Milicza. Master's thesis. Typescript. KBiER, UP Wrocław.

KĄскI Z., ed. (2003): Zagrożone gatunki flory naczyniowej Dolnego Śląska. Instytut Biologii Roślin, Uniwersytet Wrocławski, Polskie Towarzystwo Przyjaciół Przyrody „pro Natura”, Wrocław.

Kochanowska R. (1997): Przyrodnicze konsekwencje regresu gospodarki łąkowej na Pomorzu Zachodnim. Przegląd Przyrodniczy 8, 1/2: 73-76.

KondRACKI J. (1994): Geografia Polski. Mezoregiony fizyczno-geograficzne. Wyd. Nauk. PWN, Warszawa.

KowALSKA D. (1990): Tendencje sukcesyjne i wartość gospodarcza zbiorowisk roślinnych zachodniej części torfowiska niskiego koło wsi Przedmoście- 
-Święte. Master's thesis. Typescript. KBiER, UP Wrocław.

KozŁowsKa T. (2005): Zmiany zbiorowisk łąkowych na tle różnicowania się warunków siedliskowych $\mathrm{w}$ charakterystycznych obszarach dolin rzecznych Polski Centralnej. Woda - Środowisko - Obszary Wiejskie. Rozprawy Naukowe i Monografie 14.

Kryszak A., Kryszak J., Grynia M., CZemko M. (2006): Dynamika zmian różnorodności florystycznej zbiorowisk trawiastych doliny Obry. Woda - Środowisko - Obszary Wiejskie 6, 1(16): 229-237.

Kucharski L., MichalsKa-Hejduk D. (1994): Przegląd zespołów łąkowych z klasy Molinio-Arrhenatheretea stwierdzonych w Polsce. Wiadomości Botaniczne 38(1/2): 95-104.

Kucharski L., Michalska-Hejduk D., KoŁodziejek J. (2001): Przegląd zespołów torfowiskowych z klasy Scheuchzerio-Caricetea fuscae stwierdzonych $\mathrm{w}$ Polsce. Wiadomości Botaniczne 45(1/2): 33-44.

Matuszkiewicz W. (2006): Przewodnik do oznaczania zbiorowisk roślinnych Polski. Wyd. Nauk. PWN, Warszawa.

Mirek Z., Pięroś-Mirkowa H., Zając A., Zając M. (2002): Flowering plants and pteridophytes of Poland. A checklist. Vol. 1. Biodiversity of Poland. - Krytyczna lista roślin naczyniowych Polski. T. 1. Różnorodność biologiczna Polski. W. Szafer Institute of Botany, Polish Academy of Sciences, Kraków.

NoRYŚKIEWICZ A. (1978): Zbiorowiska roślinne torfowiska Zgniłka oraz zmiany zachodzące w nich pod wpływem gospodarki człowieka. Studia Societatis Scientiarum Torunensis, Sectio D (Botanica) 10, 3.

OкRuszко H. (1966): Ocena plonów siana $z$ trzech zmeliorowanych torfowisk. Wiadomości IMUZ 6, 1: 13-45.

Olaczek R., Kucharski L., Pisarek W. (1990): Zanikanie obszarów podmokłych i jego skutki środowiskowe na przykładzie województwa piotrkowskiego (zlewnie Pilicy i Warty). Studia Ośrodka Dokumentacji Fizjograficznej 18: 141-198.

Oświt J. (1996): Roślinność, wartość rolnicza i produkcyjność łąk łęgowej części doliny górnej Narwi. Zeszyty Problemowe Postępów Nauk Rolniczych 428: 51-78.

OśwIT J. (2000): Metoda przyrodniczej waloryzacji mokradeł i wyniki jej zastosowania na wybranych obiektach. Materiały Informacyjne 35 . Wyd. IMUZ, Falenty.

PAŁCZYŃSKi A. (1970): Dokumentacja geobotaniczna torfowisk (badania wstępne). Rejon: Kliczków, województwo: wrocławskie, powiat: Bolesławiec, gromady: Parowa, Osiecznica. Typescript. Wrocław.

PAŁCZYŃSKi A. (1976): Dokumentacja geobotaniczna torfowisk (badania wstępne). Rejon: Przedmoście, województwo: wrocławskie, gmina: Środa Śląska, Miękinia. Typescript. KBiER, UP Wrocław.
PAŁCZYŃSKI A. (1977): Kierunki przemian szaty roślinnej i siedlisk zatorfionych roślin dolin rzecznych pod wpływem ingerencji człowieka. Zeszyty Problemowe Postępów Nauk Rolniczych 169: 87-102.

PAŁCZYŃSKI A. (1985): Succession trends in plant communities of the Biebrza valley. Polish Ecological Studies 11, 1: 5-20.

PoDlasKa M. (2009): Zbiorowiska roślinne nieużytkowanych łąk pobagiennych na Dolnym Śląs$\mathrm{ku}$, ich przemiany oraz wartość gospodarcza i przyrodnicza. Doctoral dissertation. Typescript. KBiER, UP Wrocław.

Ротт R. (1995): Die Pflanzengesellschaften Deutschlands. Ulmer, Stuttgart.

Prończuk J. (1956): Problem degradacji łąk na terenach organogenicznych w Polsce. Zeszyty Problemowe Postępów Nauk Rolniczych 216, 2: 45-68.

Prończuk J. (1970): Metody określania jakości siedlisk oraz wartości gospodarczej łąk. In: M. Falkowski (ed.). Łąkarstwo. T. 1. Łąkoznawstwo. PWRiL, Warszawa: 293-335.

RothMALER W. (2002): Exkursionsflora von Deutschland. Gefäßpflanzen: Kritischer Band. Band 4. Spektrum, Heidelberg, Berlin.

RozPORZĄDZENIE Ministra Środowiska z dnia 9 lipca 2004 r. w sprawie gatunków dziko występujących roślin objętych ochroną. Dz.U. 2004, nr 168, poz. 1764.

RozPoRZADZENIE Ministra Środowiska z dnia 5 stycznia 2012 r. w sprawie ochrony gatunkowej roślin. Dz.U. 2012, nr 0, poz. 81.

RUTKOwsKi L. (2004): Klucz do oznaczania roślin naczyniowych Polski niżowej. Wyd. Nauk. PWN, Warszawa.

Schube T. (1903): Die Verbreitung der Gefäßpflanzen in Schlesien preussischen und österreichischen Anteils. Druck von R. Nischkovsky, Breslau.

SoвCZAK E. (1982): Zróżnicowanie szaty roślinnej na tle warunków glebowych i przydatność gospodarcza torfowiska „Piaski”. Master's thesis. Typescript. KBiER, UP Wrocław.

StePA T. (1976): Dokumentacja geobotaniczna torfowisk (badania wstępne). Rejon: Lutynia, województwo: wrocławskie, gmina: Miękinia. Typescript. KBiER, UP Wrocław.

SzAFer W. (1972): Szata roślinna Polski Niżowej. In: W. Szafer, K. Zarzycki (eds). Szata roślinna Polski. T. 2. PWN, Warszawa: 17-188.

SzTYBER J. (2004): Analiza zmian typów siedliskowych użytków zielonych na zmeliorowanym obiekcie w dolinie rzeki Wkry. Zeszyty Naukowe Akademii Rolniczej w Krakowie 412, Inżynieria Środowiska 25: 89-98.

Toвolski K. (2003): Torfowiska na przykładzie Ziemi Świeckiej. Towarzystwo Przyjaciół Dolnej Wisły, Świecie.

ToKARsKa-Guzik B., DajDok Z. (2004): Rośliny obcego pochodzenia - udział i rola w szacie roślinnej 
Opolszczyzny. In: A. Nowak, K. Spałek (eds). Ochrona szaty roślinnej Śląska Opolskiego. Wyd. Uniwersytetu Opolskiego, Opole: 277-303.

Tomaszewska K., Podlaska M. (2007): Waloryzacja przyrodnicza zdegradowanej łąki pobagiennej. Annales Silesiae 35: 5-10.

URBAn D., GrzywnA A. (2003): Zbiorowiska roślinności łąkowej klasy Molinio-Arrhenatheretea w dolinie Ochoży. Annales Universitatis Mariae Curie-Skłodowska, Sectio E, 58: 155-166.

Weber E., Gut D. (2004): Assessing the risk of potentially invasive plant species in central Europe. Journal for Nature Conservation 12: 171-179.

WoŁejKo L. (2000): Roślinność łąkowa i ziołoroślowa $\mathrm{z}$ klasy Molinio-Arrhenatheretea kompleksów źródliskowych Polski północno-zachodniej. Folia Universitatis Agriculturae Stetinensis 213, Agricultura 85: 267-296.
Zając A., Zając M., eds (2001): Atlas rozmieszczenia roślin naczyniowych w Polsce. Pracownia Chorologii Komputerowej Instytutu Botaniki Uniwersytetu Jagiellońskiego, Kraków.

ZaŁuski T., KamiŃsKa A. (1999): Rola rowów melioracyjnych jako refugiów flory torfowiskowej na przykładzie kompleksu łąk w Koszelewkach. Folia Universitatis Agriculturae Stetinensis 197, Agricultura 75: 373-376.

Zarzycki K., Trzcińska-Tacik H., Różański W., SzeląG Z., Wotek J., Korzeniak U. (2002): Ecological indicator values of vascular plants of Poland. Vol. 2. Biodiversity of Poland. - Ekologiczne liczby wskaźnikowe roślin naczyniowych Polski. T. 2. Różnorodność biologiczna Polski. W. Szafer Institute of Botany, Polish Academy of Sciences, Kraków. 